\title{
A Direct Adaptive MNN Control Method for Stage Having Paired Reluctance Linear Actuator with Hysteresis
}

\author{
Yu-Ping Liu ${ }^{1}$, Kang-Zhi Liu ${ }^{1}$, Xiaofeng Yang ${ }^{2}$ \\ ${ }^{1}$ Department of Electrical and Electronic Engineering, Chiba University, Chiba, Japan \\ ${ }^{2}$ Department of Microelectronics, Fudan University, Shanghai, China \\ Email: eric.liuyuping@gmail.com, kzliu@faculty.chiba-u.jp, xf yang@fudan.edu.cn
}

Received 28 August 2014; revised 20 September 2014; accepted 6 October 2014

Copyright @ 2014 by authors and Scientific Research Publishing Inc.

This work is licensed under the Creative Commons Attribution International License (CC BY). http://creativecommons.org/licenses/by/4.0/

c) (i) Open Access

\begin{abstract}
Reluctance linear actuator, which has a unique property of small volume, low current and can produce great force, is a very promising actuator for the fine stage of the next-generation lithographic scanner. But the strong nonlinearities including the hysteresis, between the current and output force limits the reluctance linear actuator applications in nanometer positioning. In this paper, a new nonlinear control method is proposed for the stage having paired reluctance linear actuator with hysteresis using the direct adaptive neural network, which is used as a learning machine of nonlinearity. The feature of this method lies in that the nonlinear compensator in conventional methods, which computed the current reference from that of the input and output force is not used. This naturally overcomes the robustness issue with respect to parameter uncertainty. Simulation results show that the proposed method is effective in overcoming the nonlinearity between the input current and output force and promising in precision stage control.
\end{abstract}

\section{Keywords}

Direct Adaptive MNN, Reluctance Linear Actuator, Hysteresis

\section{Introduction}

In integrated circuit (IC) manufacturing, lithographic scanner uses reticle and wafer stage based on a two layer coarse and fine structure [1] to achieve high acceleration and high precision during quick synchronous scanning. The coarse stage moves in long stroke with high acceleration to realize micrometer level positioning precision while fine stage moves in a short stroke to realize nanometer-level positioning precision. Therefore, the fine 
stage is playing the key role in lithography.

The more functionality is packed into each IC, the smaller feature size indicative of the smallest component that can be manufactured in one IC is required. Today, this minimum feature size is about [2], and in a foreseeable future it will be smaller. And the chip manufacturers continually increase the productivity in such demanding nanoprecision conditions. The high speed, high acceleration and nanopositioning precision requirements make the lithographic scanner challenging from a position control perspective. Due to its low efficiency and high power dissipation, the voice coil actuator is no longer the best choice as the main driving actuator for the next-generation fine stage [3].

Since the reluctance linear actuator can provide a greater force with a small volume and low power gain than the voice coil actuators, it can provide a solution for driving the fine stage [4]. However, the reluctance linear actuator has a strong nonlinearity between the input current and output force, so we need to study the control method to obtain a predictable force for the requirements in nanopositioning accuracy. Conventionally, the positioning control design of a stage falls into two parts: 1) the actuator dynamics is omitted and the force is designed; 2) the current reference is computed from the designed force based on their static nonlinear relation [5]. But it does not consider the effect of hysteresis and parameter uncertainty [6] on the force accuracy. For the hysteresis compensation, using the inverse hysteresis model [7] is the most noticeable. Reference [8] proposed an inverse hysteresis model and obtained a good performance for the reluctance linear actuator. However, the above methods both need precise hysteresis model, which is generally complex and hardly to obtain.

Owing to its online self-learning ability, the neural network provides a good solution for solving nonlinear problems. Especially, the multi-layer neural network (MNN) is effectively used in nonlinear discrete-time system identification and control [9] [10]. Although a lot of researches have been done on the neural network application to the hysteresis [11] [12], there is few hysteresis compensation algorithms for the reluctance actuator from the available information. A hysteresis compensation configuration for the current-driven reluctance actuator with hysteresis using adaptive MNN has been proposed in paper [13] by the authors, but this method has to use the reluctance actuator model.

In this paper, a new and direct adaptive MNN [10] controller is proposed for the current-driven reluctance linear actuator with hysteresis. The main advantage of the proposed adaptive MNN controller is that the reluctance linear actuator model and the inverse hysteresis model are not required. Then, a control configuration is proposed for stages having paired E/I core actuator with hysteresis using the proposed adaptive MNN controller. Simulations are conducted on a stage having paired reluctance actuator with hysteresis and the results show that the adaptive MNN controller is effective in overcoming the nonlinearity and parameter uncertainty of the reluctance linear actuator.

\section{Parametric Hysteresis Operator}

Hysteresis is a typical nonlinearity encountered over a wide range of applications. The hysteresis can be briefly defined as a loop in the input-output map. In this section, the parametric hysteresis operator [8] is reviewed, which is defined in the discrete time domain for the ease of implementation.

Definition 1. Let $r(k), y(k)$ be bounded, $\lambda_{1}, \lambda_{2}>0$. Then the parametric hysteresis operator

$$
y(k)=H_{\text {hys }}\left[r(k), \lambda_{1}, \lambda_{2}\right]
$$

is defined as follows: when $r(k+1)-r(k) \geq 0$,

$$
y(k)=r(k)+y_{0}-r_{0}+m-\frac{1}{\lambda_{2}} W\left(\lambda_{2} m \cdot e^{\lambda_{2}\left(m+r(k)-r_{0}\right)}\right)
$$

when $r(k+1)-r(k)<0$,

$$
y(k)=r(k)+y_{0}-r_{0}-n+\frac{1}{\lambda_{2}} W\left(\lambda_{2} n \cdot e^{\lambda_{2}\left(n-r(k)+r_{0}\right)}\right)
$$

where $m=\lambda_{1}+r_{0}-y_{0}$ and $n=\lambda_{1}-r_{0}+y_{0}, r_{0}=r\left(k^{*}\right)$ and $y_{0}=y\left(k^{*}\right)$. The indicator $k^{*}$ denotes the last time instant before $k$ when the difference of $r$ changed sign, i.e. an extremum occurred, which corresponds to a corner point of the $y-r$ curve in Figure 1. The parameter $\lambda_{1}$ represents the amount of hysteresis around the straight line $r(k)=y(k)$ and defines the asymptotes, while the parameter $\lambda_{1}$ defines the smoothness of 

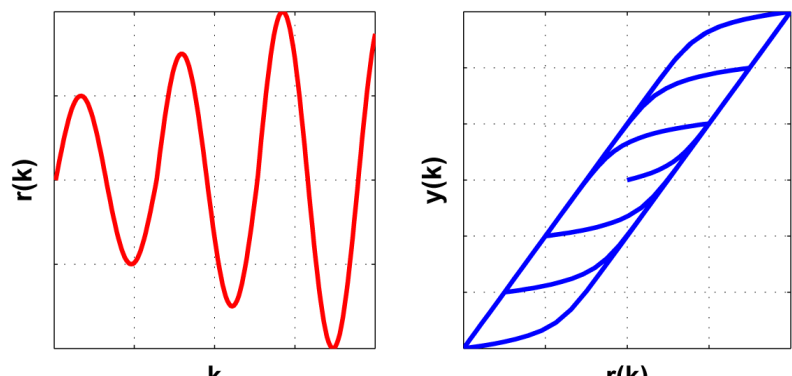

$\mathbf{r}(\mathbf{k})$

Figure 1. Input $r(k)$ (left) and the corresponding output $y(k)$ map (right)

trajectories of the parametric hysteresis operator.

the hysteresis loops, i.e. the rate of convergence towards the left or right asymptote. $W$ is the principal branch of the Lambert $W$ function [14].

\section{Reluctance Actuator Models}

Reluctance linear actuator is a type of electric motor that induces non-permanent magnetic poles on the ferromagnetic rotor and produces temporary magnetic. The reluctance actuator is named as such because it uses magnetic reluctance to generate force, which can be called reluctance force. The E/I core actuator is a most basic linear reluctance linear actuator [15]. As shown in Figure 2, the actuator includes a generally Cobalt-Iron E shaped electromagnet and an I shaped target. The electromagnet has an electrical coil wound around the center section. Current flowing through the coil generates a magnetic flux and this flux creates a reluctance force on the target. The amount of current determines the amount of reluctance force. The reluctance force $F$ acting on the $I$ target is described by [15]:

$$
F=\eta \frac{i^{2}}{x_{g}^{2}}
$$

where $\eta=\frac{\mu_{0} N^{2} A}{4}$ is the electromagnetic constant, $\mu_{0}$ is the permeability of air, $N$ is the number of turns in coil on the center leg of the E-core and $A$ is the area of the air gap. It is a lumped model which disregards unmodeled effects such as hysteresis leakage, fringing and saturation.

However, the E-core coil uses the soft magnetic material, which has magnetic hysteresis [6] between the magnetic field $H$ and the bulk magnetic flux density $B$. It is shown that the hysteretic B-H can be modeled [9] by the parametric hysteresis operator Equation (1). Then we get a reluctance linear actuator model with hysteresis as:

$$
F=\eta \frac{\left[H_{h y s}\left(i, \lambda_{1}, \lambda_{2}\right)\right]^{2}}{x_{g}^{2}}
$$

This model contains both the hysteresis and the quadratic relation between the input current $i$ and output force $F$.

\section{Nonlinear Control Using Adaptive Neural Network}

Figure 3 shows a typical feedback control loop for a current-driven reluctance linear actuator, in which "C" denotes a linear position controller. The position controller uses the air gap $x_{g}$ measured by a position sensor to generate the force command $F_{d}$. The power amplifier supplies the current for the reluctance linear actuator. From Equation (4), the current command is obtained [5] as:

$$
i_{d}=\sqrt{\frac{F_{d} \cdot x_{g}^{2}}{\eta_{d}}} s
$$




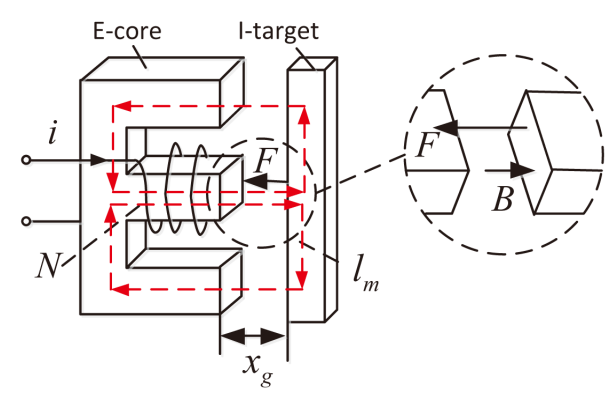

Figure 2. Sketches of the E-core actuator.

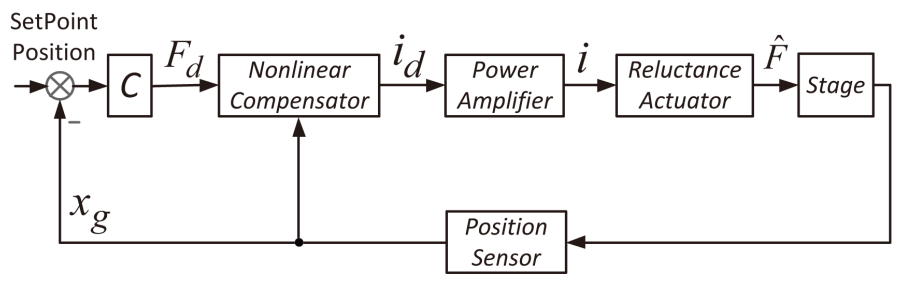

Figure 3. Shapes of an input current and the corresponding output force based on the reluctance actuator model with hysteresis.

where $\eta_{d}$ is the estimate of real constant $\eta$. But the current compensator Equation (6) does not consider the hysteresis in the reluctance force and is highly sensitive to the parameter $\eta_{d}$. However, hysteresis effect must be considered for high-precision motion control. In the following, a direct hysteresis compensator for the reluctance actuator based on the adaptive MNN is proposed.

It is well known that in control engineering, artificial NN can be used as a universal function emulator. MNN is a static feed-forward network that consists of a number of layers, each layer having of number of McCullochPitts neurons. Due to its hidden layers, the MNN can approximate any continuous nonlinear function. Once the hidden layers have been selected, only the adjustable weights have to be determined to specify the networks completely. When the weights are updated online, the network is called adaptive MNN.

A nonlinear controller using the adaptive MNN [10] for the current-driven reluctance actuator is proposed as shown in Figure 4. This structure has the advantages is that study the nonlinearity between the force command $F_{d}$ and the output force $F$ and overcomes the robustness issue with respect to parameter uncertainty. The feedback force $F$ can be estimated from the relation $F=m a$, with $m$ the stage mass and $a$ the actual acceleration. The actual acceleration $a$ can be measured by an accelerometer or computed from the measured gap position $x_{g}$ by a digital double differentiator. The double differentiation may introduce a noise. However, when using a filter such as the least-square method and considering the noise level, it makes double differentiation acceptable as a method to calculate the stage acceleration [16]. In this article, the acceleration $a$ is measured by the accelerometer. The detail of the adaptive MNN is as follows.

At instant $k$, the command current $i_{d}(k)$ is given by:

$$
i_{d}(k)=\mathbf{W}^{\mathrm{T}}(k) \xi\left(\mathbf{V}^{\mathrm{T}}(k) \overline{\mathbf{p}}(k)\right)
$$

where $\mathbf{W}(t)=\left[w_{1}, \cdots, w_{l+1}\right] \in \mathbf{R}^{l+1}$ and $\mathbf{V}(t)=\left[v_{1}, \cdots, v_{l}\right] \in \mathbf{R}^{(n+1) \times l}$ are weighting matrices, $l$ is the number of hidden-layer neurons, select $\mathbf{p}(k)=\left[F_{d}(k+1), F_{d}(k), F(k+1), F(k)\right]$, then $\overline{\mathbf{p}}(k)=[\mathbf{p}(k), 1]^{\mathrm{T}}$ denotes the neural network input, $\xi\left(\mathbf{V}^{\mathrm{T}} \overline{\mathbf{p}}(k)\right)=\left[\xi\left(v_{1}^{\mathrm{T}} \overline{\mathbf{p}}(k)\right), \cdots, \xi\left(v_{l}^{\mathrm{T}} \overline{\mathbf{p}}(k)\right), 1\right]^{\mathrm{T}}$ with $\xi(p)=1 /\left(1+\mathrm{e}^{-p}\right)$. The force tracking error is:

$$
\varepsilon(k+1)=F_{d}(k)-F(k)
$$

The neural network weights are updated online based on the adaptive laws using the force tracking error Equation (8). The adaptive update laws are chosen as 


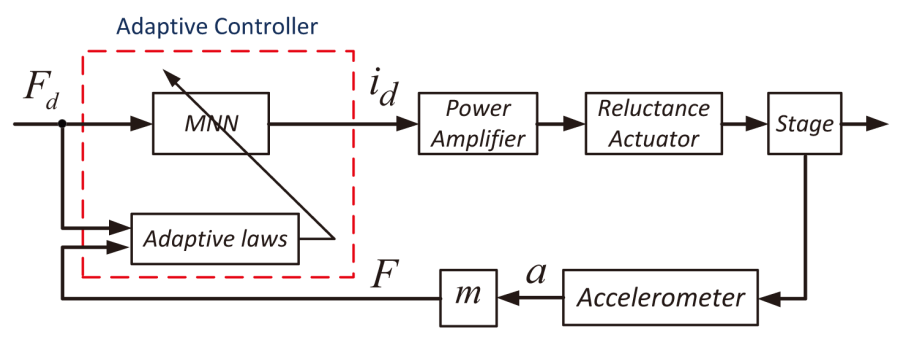

Figure 4. Direct adaptive MNN control for reluctance linear actuator.

$$
\begin{gathered}
\mathbf{W}(k+1)=\mathbf{W}(k)-\operatorname{Proj}_{\hat{W}}\left[\Gamma_{w} \xi(k) \varepsilon(k+1)\right] \\
\mathbf{V}(k+1)=\mathbf{V}(k)-\operatorname{Proj}_{\mathbf{V}}\left[\Gamma_{v} \mathbf{z}_{l} \mathbf{W}^{\mathrm{T}}(k) \xi^{\prime}(k) \varepsilon(k+1)\right]
\end{gathered}
$$

where $\gamma_{w}$ and $\gamma_{v}$ are the positive learning rates, $\xi(k)=\operatorname{diag}\left\{\xi_{1}(k), \cdots, \xi_{l}(k)\right\}$ with $\xi_{i}(k)=\xi\left(v_{i}^{\mathrm{T}} \overline{\mathbf{p}}(k)\right)$, $\mathbf{z}_{l}=[1 / \sqrt{l}, \cdots, 1 / \sqrt{l}]^{\mathrm{T}}(\|\mathbf{z}\|=1)$ is a vector compatible with $\mathbf{V}(k) \cdot \xi^{\prime}(k)=\operatorname{diag}\left\{\xi_{1}^{\prime}(k), \cdots, \xi_{l}^{\prime}(k)\right\}$ is a diagonal matrix with $\xi_{1}^{\prime}(k)=\xi^{\prime}\left(v_{i}^{\mathrm{T}} \overline{\mathbf{p}}(k)\right)$. Since the output of the hidden layer neural network is directly related to the input of the output weight, so the output weight $\mathbf{W}$ is introduced in Equation (10). Define the projection function $\operatorname{Proj}_{\theta}(*)=\left\{\operatorname{Proj}_{\theta}\left(*_{j k}\right)\right\}$, whose element in the $j$-th row and $k$-th column is:

$$
\operatorname{Proj}_{\theta}\left(*_{j k}\right)=\left\{\begin{array} { c } 
{ - * _ { j k } } \\
{ * _ { j k } }
\end{array} \text { if } \left\{\begin{array}{c}
\theta_{j k}=\rho_{\theta_{j k}, \max } \text { and } *_{j k}<0 \\
\theta_{j k}=\rho_{\theta_{j k}, \min } \text { and } *_{j k}>0, \\
\text { otherwise }
\end{array}\right.\right.
$$

where $*$ is either a vector or matrix with its element being $*_{j k}, \rho_{\theta_{j k} \text {, min }}$ and $\rho_{\theta_{j k} \text {, max }}$ are presumed upper and lower boundaries of $\theta_{i j}$, and $\theta$ denotes $\mathbf{W}$ and $\mathbf{V}$. However, due to the fact that those bounds may not be known a prior, certain fictitious large enough bounds can be used [17]. It is just need to limit the output amplitude of the output of adaptive MNN Equation (7), e.g. corresponding the power amplifier input limit $\pm 10 \mathrm{v}$.

\section{Application to 1-DOF Stage Having Paired Reluctance Linear Actuator}

Due to the nature of reluctance force, an E/I core actuator can only generate a unidirectional attractive force. To generate an active force in the opposite direction, a second actuator needs to be placed on the opposite side. Figure 5 illustrates a simplified a stage [4] having electromagnetic actuators E-core 1 and E-core 2. The gaps between the E-core 1, 2 and stage are $x_{g 1}$ and $x_{g 2}$, which can be measured by suitable sensors such as capacitor sensor. The force $F_{1}$ and $F_{2}$ are nonnegative, while the difference between $F_{1}$ and $F_{2}$ can take any value and direction. In the initial state, the gaps are $x_{g 1}=x_{g 0}$ and $x_{g 2}=x_{g 0}$, where $x_{g 0}$ is the initial gap. In Figure 5, we define the right as the positive direction. Furthermore, when the stage moves to the positive direction, the gaps become $x_{g 1}=x_{g 0}-x$ and $x_{g 2}=x_{g 0}+x$ respectively where $x$ is the global placement and can be measured by position sensor such as a laser interferometer [4]. A control loop for one degree of freedom stage having paired E/I core actuator using adaptive MNN is shown in Figure 6. Since two reluctance actuators are used, we need two adaptive MNN controllers to compute the desired current for each of them.

In Figure 6, the controller $C$ uses the position error between the set-point and the measured position to determine the desired force $F_{d}$ which should be exerted to reach desired stage position. The force distributor distributes the desired force to each E/I actuator as follows:

$$
\begin{aligned}
& F_{d 1}=F_{0}+F_{d} / 2 \\
& F_{d 2}=F_{0}+F_{d} / 2
\end{aligned}
$$




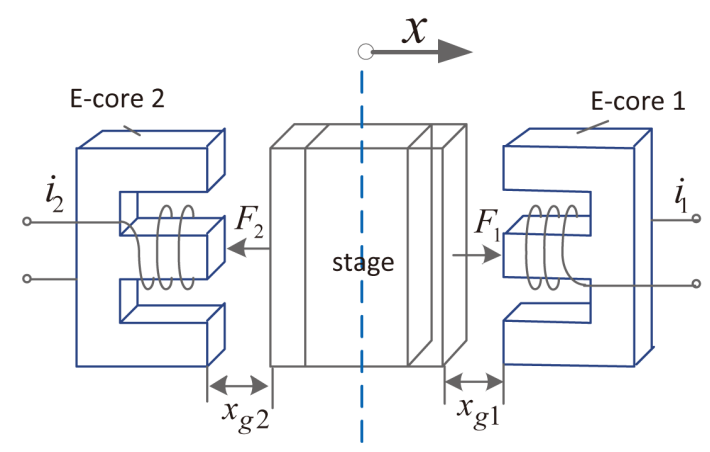

Figure 5. Paired E/I core actuator.

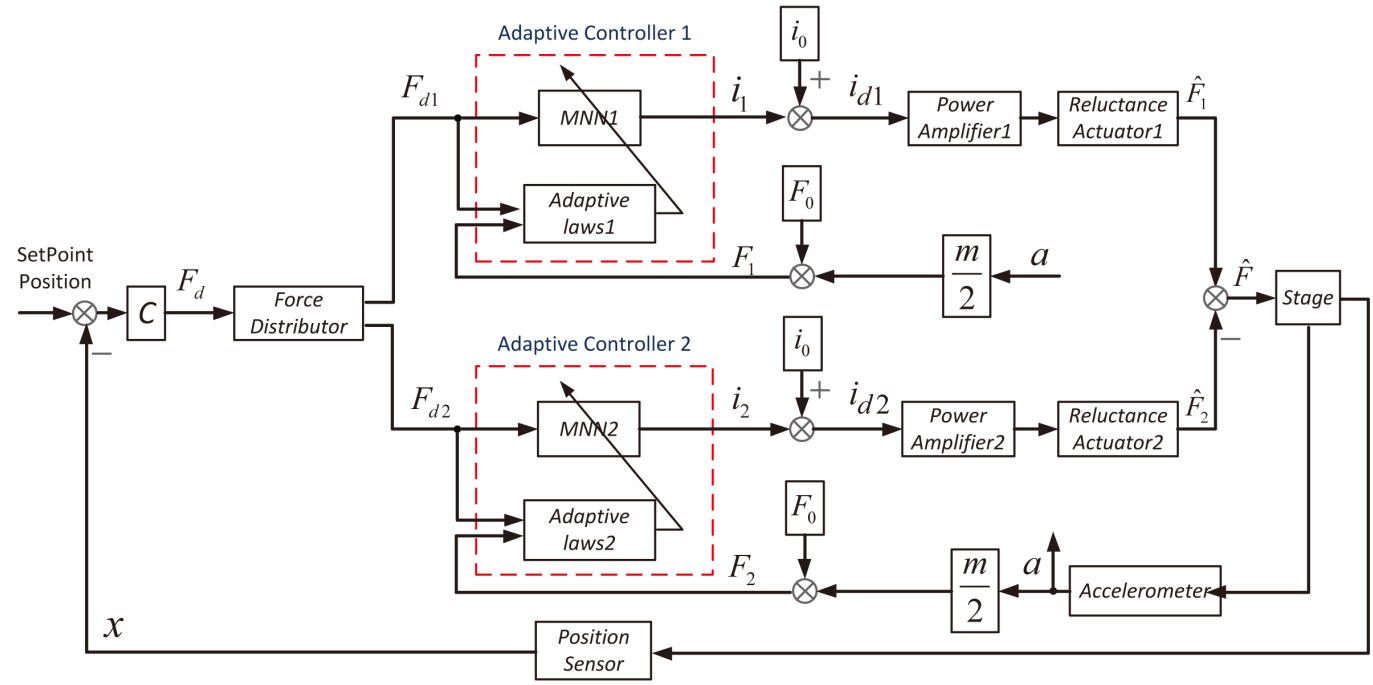

Figure 6. Nonlinear control structure for stage having paired E/I core actuator using adaptive MNN.

where $F_{0}$ is the bias force generated by paired $\mathrm{E} / \mathrm{I}$ actuator respectively in the opposite direction, which provides a zero net force on the stage, thus maintaining the position of the stage. In this paper, $F_{0}$ is determined as $F_{0}=F_{\max } / 2$, in which $F_{\max }$ is the maximum force corresponding the maximum acceleration. More details about how to select the bias force $F_{0}$ can be found in patent [6]. The feedback force $F_{1}$ and $F_{2}$ are defined as follows:

$$
\begin{aligned}
& F_{1}=F_{0}+\frac{m a}{2} \\
& F_{2}=F_{0}-\frac{m a}{2}
\end{aligned}
$$

Following Equation (8), the force tracking errors are defined as follows:

$$
\begin{aligned}
& \varepsilon_{1}(k+1)=F_{d 1}(k)-F_{1}(k) \\
& \varepsilon_{2}(k+1)=F_{d 2}(k)-F_{2}(k)
\end{aligned}
$$

The currents $i_{1}$ and $i_{2}$ with adaptive MNN controller are computed from Equation (6) and the corresponding network weights Equation (9) and Equation (10), which are updated by the force tracking errors Equation (16) and Equation (17). Then the desired currents $i_{d 1}$ and $i_{d 2}$ are obtained as follows:

$$
\begin{aligned}
i_{d 1} & =i_{1}+i_{0} \\
i_{d 2} & =i_{2}+i_{0}
\end{aligned}
$$


where current $i_{0}$ corresponds to the bias force $F_{0}$ and can be determined experimentally.

The real net force $\hat{F}$ acting on the stage is the difference between the real forces $\hat{F}_{1}$ and $\hat{F}_{2}$ :

$$
\hat{F}=\hat{F}_{1}-\hat{F}_{2}
$$

\section{Simulations}

Example 1: A simulation for the current-driven reluctance linear actuator is conducted to verify the performance of the proposed adaptive MNN control configuration shown in Figure 4. For comparison the nonlinear compensator Equation (6) is selected.

In this simulation, since the linear power amplifier has a high bandwidth and rapid response, it is ignored. A force command $F_{d}$ is imposed without a counter balance force, the I-target would be pulled to the E-core. For this reason, it is assumed that the I-target is fixed, so that we may investigate the force tracing performance. The reluctance linear actuator with hysteresis is modeled by Equation (5), which parameters are chosen as in the patent [15] the maximum force is about $200 \mathrm{~N}$, the maximum gap $x_{g}$ between the E-core and I-target is $0.4 \mathrm{~mm}$ and the constant $k=7.73 \times 10^{-6}$. Here, the hysteresis operator parameters are chosen as $\lambda_{1}=0.02$ and $\lambda_{2}=10$.

The control objectives is to make the system output $F$ to follow the reference force $F_{d}$. The adaptive MNN controller Equation (7) is designed to reduce the influence of the nonlinearity including the hysteresis. Parameters of the adaptive MNN are determined as follows: the hidden-layer neurons are $l=40$, the initialization neural network weighting matrices are $\mathbf{W}(0)=0$ and $\mathbf{V}(0)=0$. Equation (9) and Equation 10 are used to update the neural network weights, and the learning rate are selected as $\gamma_{w}=0.00085$ and $\gamma_{v}=0.01$.

The force tracking error between the input $F_{d}$ and output $F$ with nonlinear controller Equation (6) is shown in Figure 7. When $\eta_{d}=\eta$, the force tracking error is about $\pm 4 \mathrm{~N}$; when $\eta_{d}=0.95 \eta$, force tracking error is about from $-12.5 \mathrm{~N}$ to $0.5 \mathrm{~N}$; when $\eta_{d}=1.05 \eta$, force tracking error is about from $-0.5 \mathrm{~N}$ to $11 \mathrm{~N}$. It can be seen that the force error increases with the error of $\eta_{d}$ increases quickly. The force tracking error with adaptive MNN controller Equation (6) is shown in Figure 8. The force tracking error is about $\pm 0.25 \mathrm{~N}$ even if the E/I model parameter $\eta$ varies up $\pm 20 \%$. The reason is that the current command $i_{d}$ is determined independent of the force model Equation (4). Furthermore, Figure 9 shows that the ratio between the output force and its command is very close to 1 with adaptive controller. For comparison the nonlinear compensator Equation (6) with parameter $\eta_{d}=\eta$ is selected. From the simulation results, it can be seen that the force with adaptive MNN control has a better tracking performance and robustness against parameter uncertainty than with nonlinear controller. This paper also provides a technical solution in other precision machine tools and related equipment with hysteresis.

Example 2: In order to verify whether the proposed hysteresis compensation using adaptive MNN controller Equation (7) can be applied in high-precision systems, we do the following simulation on the one degree of freedom stage as shown in Figure 10. For comparison the nonlinear compensator Equation (6) is used.

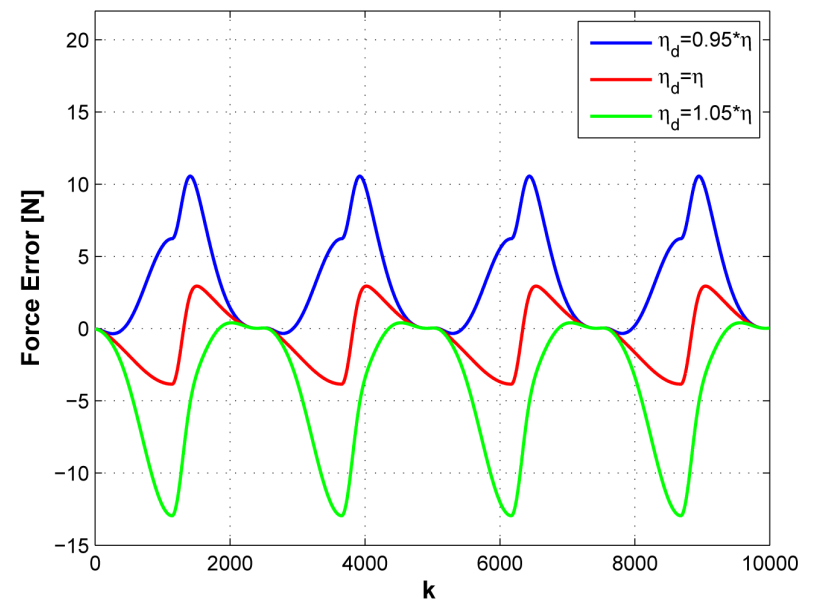

Figure 7. Force error with nonlinear compensator. 


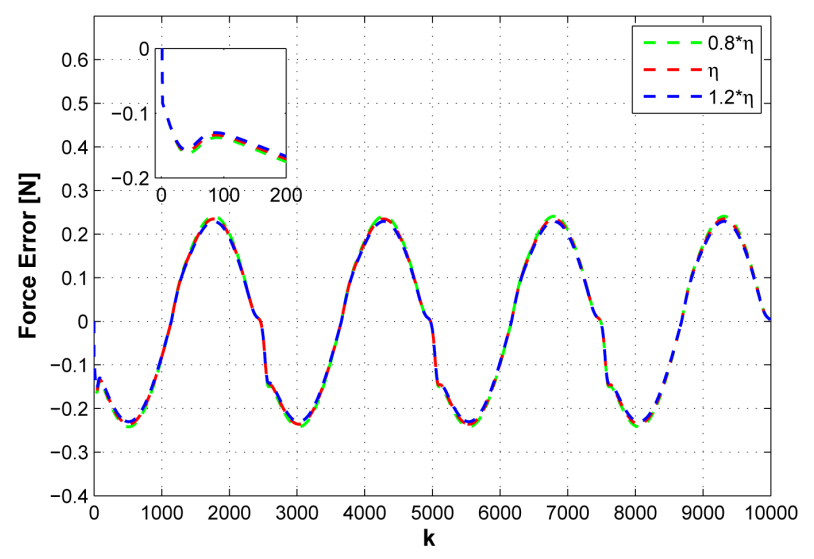

Figure 8. Force error with adaptive MNN controller.

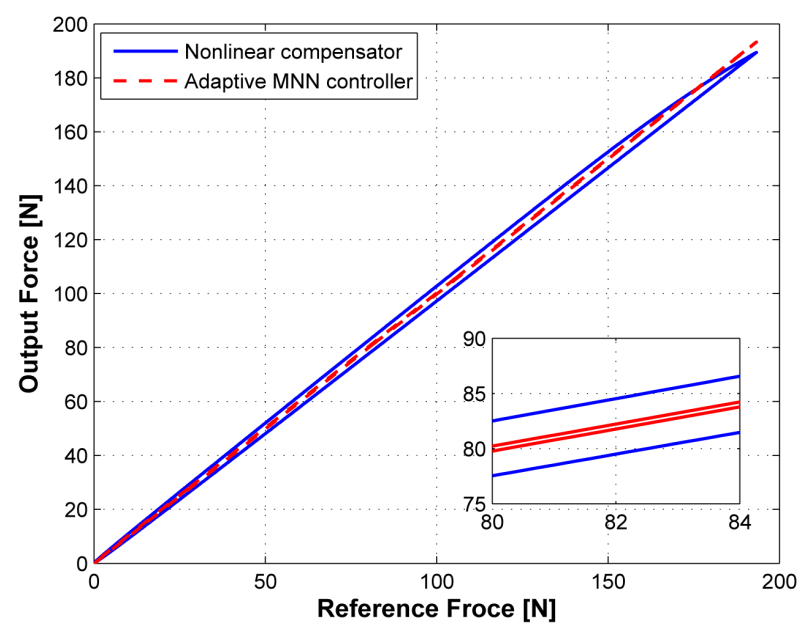

Figure 9. Comparison of the force hysteresis loop between nonlinear compensator and adaptive controller.

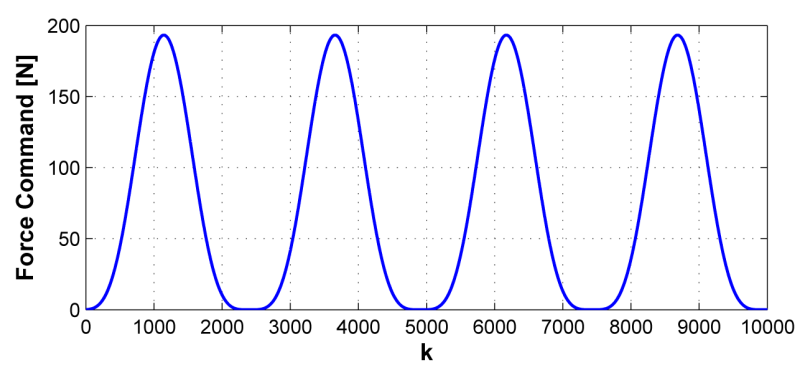

Figure 10. Force command.

The stage mass is $10 \mathrm{~kg}$ and the bias force is $F_{0}=15 \mathrm{~N}$, which is applied on both EI actuators. The gaps $x_{g 1}$ and $x_{g 2}$ between the E-core 1, 2 and the stage are in the range of $0 \mu \mathrm{m}$ to $400 \mu \mathrm{m}$ and the initial gap is $x_{g 0}=400 \mu \mathrm{m}$. The E-core electromagnetic constant and parameters of hysteresis operator are set as in Example 1. The adaptive MNN parameters for the two E/I actuators are the same as in Example 1. The control objective is to make the stage position $x$ follow the reference position. The reference trajectory is determined by the 3rd order trajectory planning method [18]. The position and velocity are shown in Figure 11. The largest displacement is $350 \mu \mathrm{m}$ and the maximum velocity is $10 \mathrm{~mm} / \mathrm{s}$. The maximum acceleration is $1 \mathrm{~m} / \mathrm{s}^{2}$ and the jerk is $200 \mathrm{~m} / \mathrm{s}^{3}$.

The position tracking errors of control with nonlinear compensator Equation 6 and with adaptive MNN 
controller Equation (7) are shown in Figure 12. We define the constant velocity settling time and the stop time as the time instants after which the position tracking error is less than $1 \mu \mathrm{m}$. From the constant velocity segment, it can be seen that the constant velocity settling time with nonlinear compensator $(0.0198 \mathrm{~s})$ is about 1.22 times longer than of adaptive MNN controller $(0.0162 \mathrm{~s})$. From the stop segment, the stop time with nonlinear compensator $(0.055 \mathrm{~s})$ is about 1.1 times longer than that of adaptive MNN controller $(0.05 \mathrm{~s})$. The outputs of adaptive MNN controllers are shown in Figure 13 and the corresponding output force of the E/I core actuator 1, E/I core actuator 2.

\section{Conclusion}

This paper has proposed a new direct adaptive MNN controller for the reluctance linear actuator with hysteresis. The direct adaptive MNN controller gets the desired current based on the desired force and actual force using an adaptive MNN, whose weights are updated using the projection function. Then the adaptive MNN controller is applied to a stage having paired E/I core actuator. Due to the simplicity of the nonlinear control configuration, it has a wider range of applications. The simulation results show that the proposed method is effective in overcoming the hysteresis parameter uncertainty and promising in high-precision control applications.

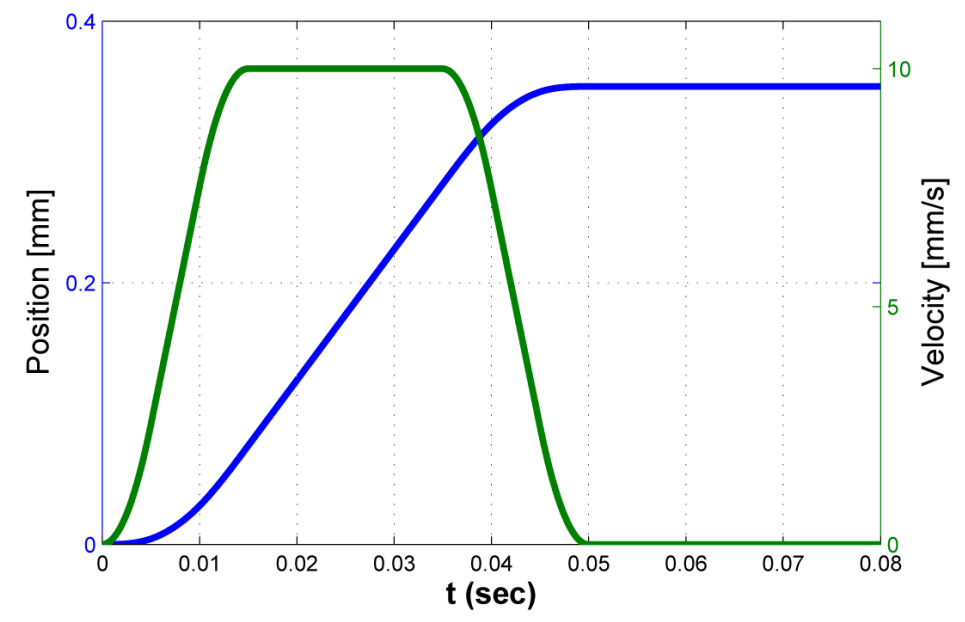

Figure 11. Reference position and velocity.

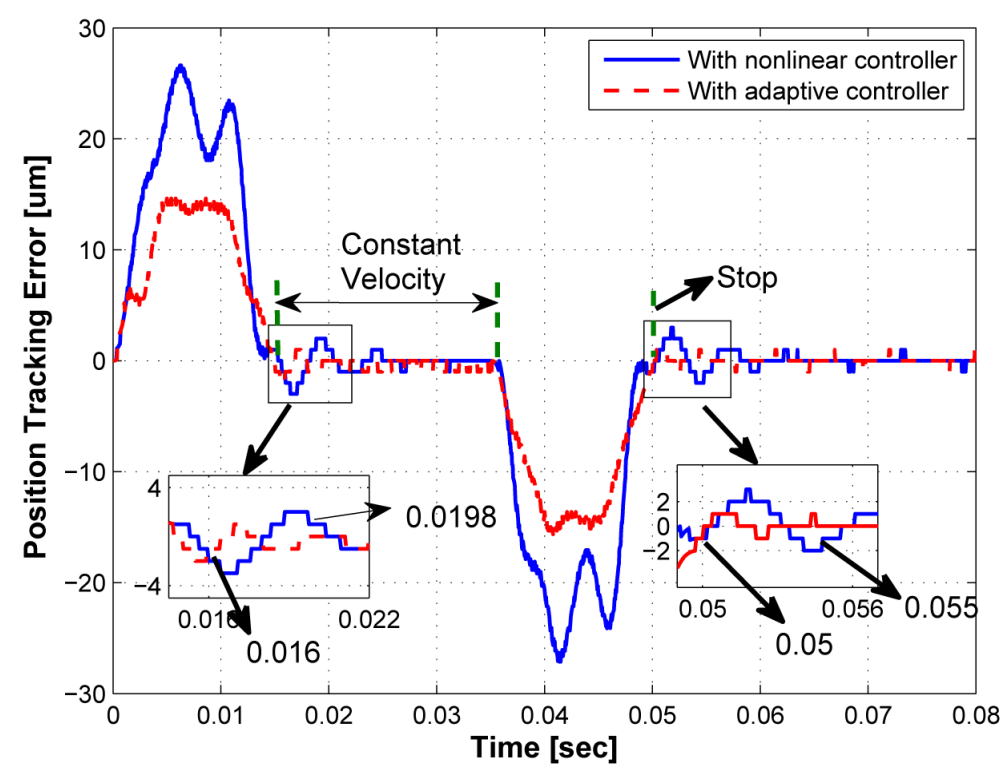

Figure 12. Comparison of the position tracking error between with nonlinear compensator and with adaptive MNN controller. 


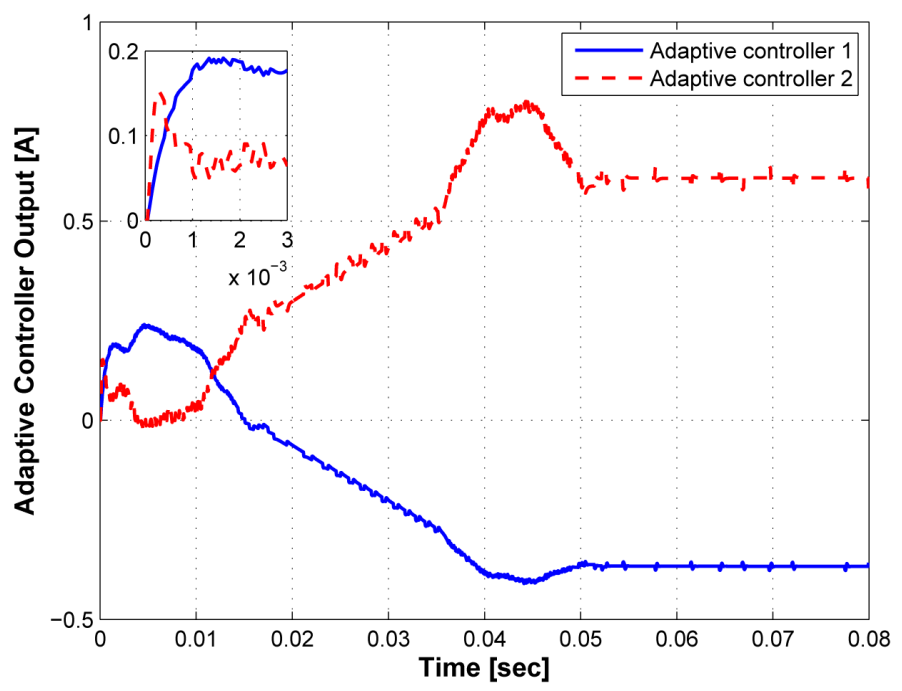

Figure 13. Outputs of the adaptive controllers.

\section{Acknowledgements}

This work is supported by National Natural Science Foundation (NNSF) of China under Grant 61274109.

\section{References}

[1] Butler, H. (2011) Position Control in Lithographic Equipment. IEEE Control Systems Magazine, 31, 28-47. http://dx.doi.org/10.1109/MCS.2011.941882

[2] Intel Technology-News (2014) Toshiba Starts Mass Production of World's First 15 nm NAND Flash Memories. http://phys.org/news/2014-04-toshiba-mass-production-world-15nm.html

[3] Vrijsen, N.H. and Jansen, J.W. (2010) Comparison of Linear Voice Coil and Reluctance Actuators for High-Precision Applications. Power Electronics and Motion Control Conference (EPE/PEMC), 2010 14th International, S3-S29.

[4] Teng, T.C. and Yuan, B. (2000) Magnetic Actuator Producing Large Acceleration on Fine Stage and Low RMS Power Gain. US Patent No. 6130517.

[5] Chang, P.-W., et al. (2007) E/I Core Actuator Commutation Formula and Control Method. US Patent No. 72535767.

[6] Bertotti, G. (1998) Hysteresis in Magnetism: For Physicists, Materials Scientists, and Engineers. Academic Press. New York.

[7] Iyer, R.V. and Tan, X. (2009) Control of Hysteretic Systems through Inverse Compensation. IEEE Control Systems, 29, 83-99. http://dx.doi.org/10.1109/MCS.2008.930924

[8] Katalenic, A. and de Boeij, J. (2011) Linearization of the Reluctance Force Actuator Based on the Parametric Hysteresis Inverse and a 2D Spline. Proceedings of the 8th International Symposium on Linear Drives for Industry Applications (LDIA 2011), Eindhoven, 3-6 July 2011.

[9] Liu, Y.P., Zhai, L. and Chai, T. (2008) Nonlinear Adaptive PID Control Using Neural Networks and Multiple Models and Its Application. Journal of Chemical Industry and Engineering (China), 59, 1671-1676.

[10] Ge, S.S., Hang, C.C., Lee, T.H. and Zhang, T. (2010) Stable Adaptive Neural Network Control. Kluwer, Boston.

[11] San, P.P., Ren, B., Ge, S.S., Lee, T.H. and Liu, J.K. (2011) Adaptive Neural Network Control of Hard Disk Drives with Hysteresis Friction Nonlinearity. IEEE Transactions on Control Systems Technology, 19, 351-358. http://dx.doi.org/10.1109/TCST.2010.2041233

[12] Lin, F.J., Shieh, H.J. and Huang, P.K. (2006) Adaptive Wavelet Neural Network Control with Hysteresis Estimation for Piezo-Positioning Mechanism. IEEE Transactions on Neural Networks, 17, 432-444.

[13] Liu, Y.P., Liu, K.Z. and Yang, X.F. (2013) Hysteresis Compensation Control for Reluctance Actuator Force Using Neural Network. The 32nd Chinese Control Conference, Xian, 26-28 July 2013, 3354-3359.

[14] Corless, R.M., Gonnet, G.H., Hare, D.E.G., Jeffrey, D.J. and Knuth, D.E. (1996) On the Lambert W Function. Advances in Computational Mathematics, 5, 329-359. http://dx.doi.org/10.1007/BF02124750

[15] Furlani, E.P. (2001) Permanent Magnet and Electromechanical Devices: Materials, Analysis, and Applications. Aca- 
demic Press, Waltham.

[16] Butler, H. (2013) Adaptive Feedforward for a Wafer Stage in a Lithographic Tool. IEEE Transactions on Control Systems Technology, 21, 875-881.

[17] Gong, J.Q. and Yao, B. (1999) Adaptive Robust Control without Knowing Bounds of Parameter Variations. Proceedings of the 38th IEEE Conference on Decision and Control, 4, 3334-3339.

[18] Roover, D. (1997) Motion Control of a Wafer Stage. Delft University Press, Delft. 
Scientific Research Publishing (SCIRP) is one of the largest Open Access journal publishers. It is currently publishing more than 200 open access, online, peer-reviewed journals covering a wide range of academic disciplines. SCIRP serves the worldwide academic communities and contributes to the progress and application of science with its publication.

Other selected journals from SCIRP are listed as below. Submit your manuscript to us via either submit@scirp.org or Online Submission Portal.
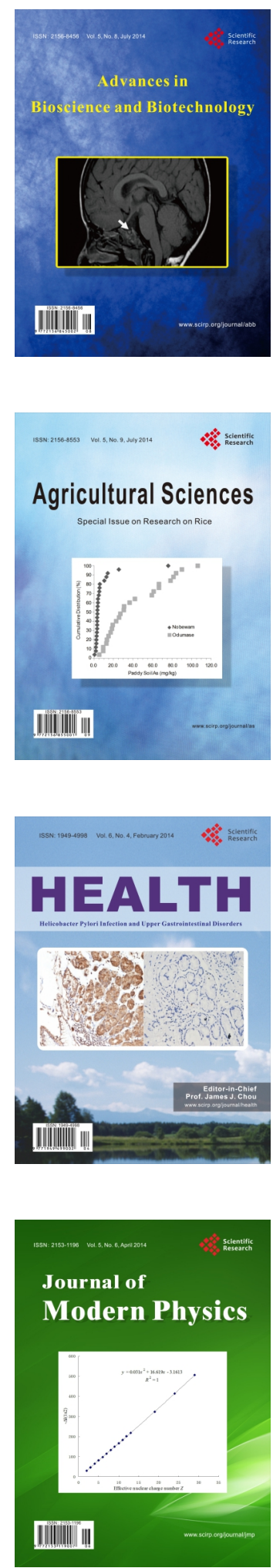
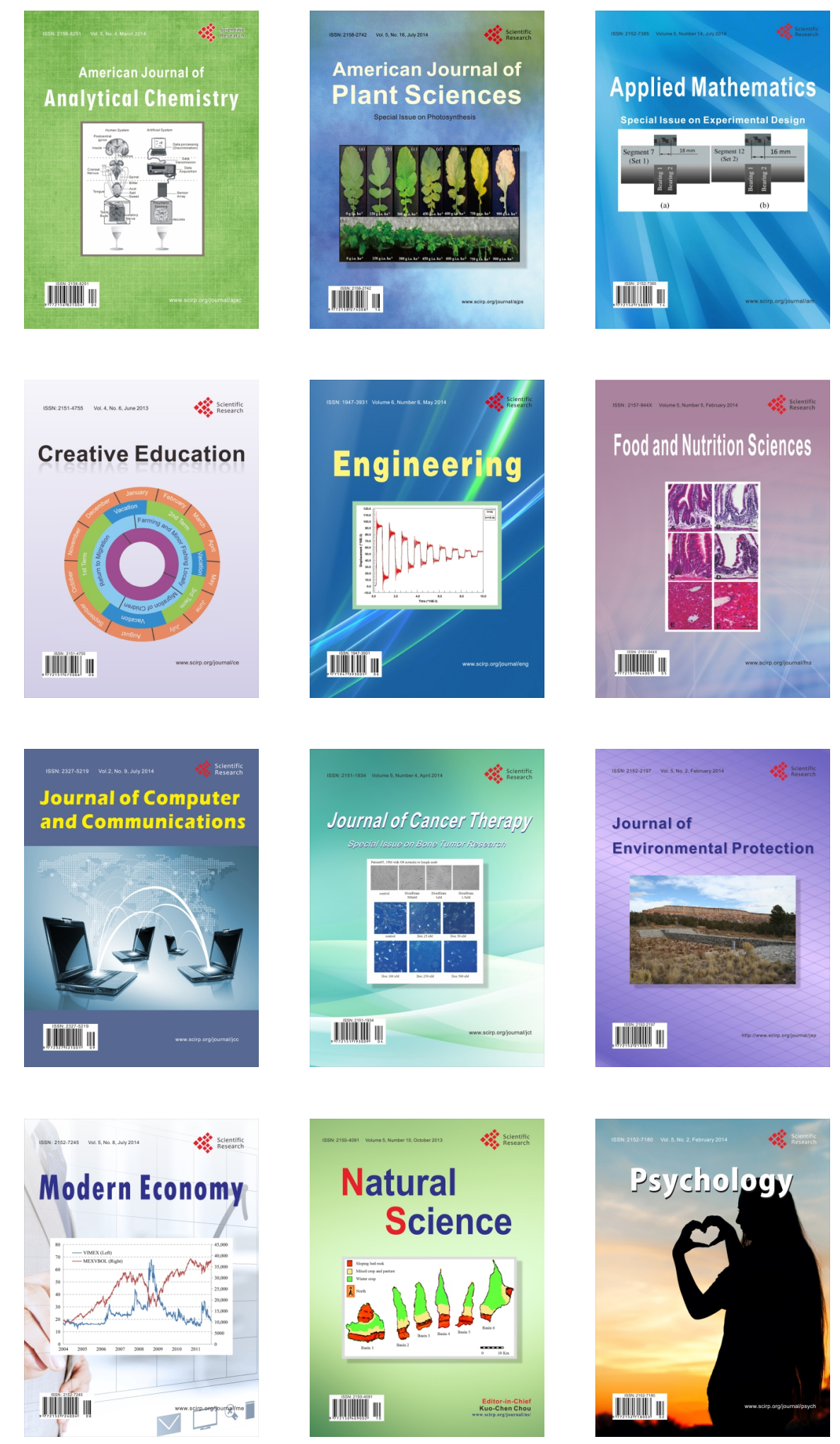